\title{
Evaluación de Competencias Transversales en Universitarios. Propiedades Psicométricas Iniciales del Cuestionario de Competencias Transversales
}

\author{
Measuring Generic Competences in College Students. \\ Psychometric Properties of the Generic Competences \\ Questionnaire
}

\author{
David Aguado ${ }^{1 *}$ \\ Antonio González ${ }^{2}$ \\ Marta Antúnez ${ }^{1}$ \\ Teresa de Dios ${ }^{3}$
}

\begin{abstract}
${ }^{1}$ Universidad Autónoma de Madrid ${ }^{2}$ CastInfo ${ }^{3}$ Universidad Francisco de Vitoria
En este trabajo se presenta el desarrollo del Cuestionario de Competencias Transversales para estudiantes Universitarios, así como sus propiedades métricas iniciales. El cuestionario se basa en las competencias identificadas por el proyecto Tuning dentro del marco del Espacio Europeo de Educación Superior. Para su desarrollo se utilizan Escalas de Observación de Conducta. En un primer estudio $(\mathrm{N}=741)$ se pone a prueba la fiabilidad de las medidas, su validez de contenido y estructura dimensional. Los resultados muestran que las escalas que componen el cuestionario tienen una fiabilidad adecuada y unos altos índices de congruencia. La estructura dimensional parece reproducir el modelo sustantivo de partida. En un segundo estudio $(\mathrm{N}=46)$ se explora la validez criterio del cuestionario frente a los resultados académicos. Los resultados muestran que diferentes competencias apreciadas por el cuestionario correlacionan significativamente con los resultados obtenidos por los alumnos en una prueba objetiva de conocimientos y los indicadores de evaluación continua. Estas competencias incrementan significativamente la varianza explicada en las calificaciones académicas por la personalidad y la inteligencia. Tomados en conjunto los datos permiten avalar la fiabilidad y validez de las medidas obtenidas con el cuestionario.
\end{abstract}

Descriptores: Evaluación del estudiante, Desarrollo de competencias, Test, Educación universitaria.

In this paper is presented the development of the Students' Generic Competences Questionnaire, as well as its initial metric properties. The questionnaire is based on the competencies identified by the Tuning project within the EEES frame. Standard Behavioral Observation Scales are used to develop it. In a first study $(\mathrm{N}=$ 741 ) is set to test the reliability of the measurements obtained, its content validity and dimensional structure. The results show that the scales making up the questionnaire have adequate reliability and high rates of consistency, and dimensional structure model seems to reproduce the starting model. In a second study $(\mathrm{N}=46)$ the criterion validity of the questionnaire versus academic results is explored. The results show that different competencies assessed by the questionnaire correlated significantly with the results obtained by students in an objective knowledge test and continuous assessment indicators. These skills significantly increase the explained variance in academic qualifications by the personality and intelligence. Together this data allows us to guarantee the reliability and validity of measurements obtained with the questionnaire.

Keywords: Student evaluation, Skills development, Testing, University education.

*Contacto: david.aguado@uam.es

ISSN: $1696-4713$

www.rinace.net/reice/

revistas.uam.es/reice
Recibido: $\quad 29$ de mayo 2016

$1^{\text {a }}$ Evaluación: 30 de agosto 2016

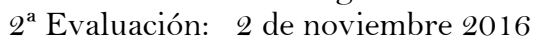

Aceptado: 12 de diciembre 2016 


\section{Revisión de la literatura}

Los Modelos de Gestión por Competencias (MGC) gozan de un importante prestigio entre los profesionales de las organizaciones (Boam y Sparrow, 1992; Feltham, 1992; Sparrow y Bognanno, 1993). Esto ha sido debido fundamentalmente a la necesidad de que los modelos de Gestión de Recursos Humanos (GRH) se adapten a las nuevas estructuras organizativas caracterizadas tanto por una mayor flexibilidad como por la emergencia de un modelo productivo soportado en el conocimiento (Nybo, 2004). La definición de qué es una competencia no ha estado exenta de controversia y desde las definiciones iniciales propuestas (Boyatzis, 1982; McClelland, 1973; Spencer y Spencer, 1993) hasta la visión actual un largo camino se ha recorrido. Actualmente se entienden las competencias como repertorios de conducta diferenciales que llevan a las personas a desarrollar con mayor eficiencia su trabajo (Sparrow, 1997) y que se componen del conjunto de comportamientos que son instrumentales para la consecución de un desempeño exitoso en el puesto (Bartram, 2005).

Los MGC han sido parte de la respuesta que desde la GRH se ha realizado frente a los cambios estratégicos generados por un modelo productivo basado en el conocimiento (Sparrow y Bognanno, 1993) en el que el compromiso y el desarrollo de carrera emergen como factores críticos para el desarrollo organizacional (Akkermans, Schaufeli, Brenninkmeijer y Blonk, 2013). Así, como indica Lawler (1994), las organizaciones que mejor se adaptan a los cambios en sus entornos de actuación son las que se basan en las competencias de sus empleados. De forma general, la gestión por competencias permite que las organizaciones clarifiquen los comportamientos y prácticas necesarias para cubrir con éxito los diferentes puestos de trabajo, ayudan a encontrar a los trabajadores cuyas competencias les aseguran un desempeño exitoso, maximizan la productividad y la eficiencia de las inversiones en formación y desarrollo, y hacen que los comportamientos de los empleados se orienten a los valores y estrategias de la organización (Lucia y Lepsinger, 1999). En cualquier caso es necesario indicar que la utilización de dichos modelos no está exenta de polémica debido fundamentalmente a los déficits en la implantación de los mismos (García, 2011).

Así, se han desarrollado diferentes trabajos para establecer modelos de competencias generales que pudieran ser aplicados a diferentes posiciones laborales (Nikolau, 2003), en lo que se ha denominado modelos de competencias transversales. Entre ellas destacan las propuestas de Tett y otros (2000), Robertson y otros (1999), Robertson y Kinder (1993) y los desarrollos de Bartram con el grupo SHL (Bartram, 2005; Kurz y Bartram, 2002).

En este contexto, no es de extrañar que las organizaciones demanden de la universidad el desarrollo de competencias transversales en sus titulados y que la Universidad no haya sido ajena a esta demanda (Palmer, Montaño y Palou, 2009). En el marco de las diferentes convenciones europeas sobre la convergencia en el Espacio Europeo para la Educación Superior (EEES), se desarrolló el proyecto Tuning Education Structures in Europe (González y Wagenaar, 2003), en el que, entre otras cosas, se definen un conjunto de competencias transversales que los universitarios deben adquirir complementariamente a sus habilidades técnicas. Más recientemente diferentes trabajos han tratado de establecer de entre dichas competencias, aquellas que son clave para el futuro que la Unión Europea ha de afrontar en el medio plazo. Desde Tuning se establece que la forma de desarrollar las competencias necesarias en los universitarios es alinear el 
perfil del titulado universitario con el que académicos, empleadores y agentes profesionales demandan. Fruto del análisis realizado en el marco de Tuning se identificaron treinta competencias genéricas (transversales) (ver tabla 1) agrupadas en tres categorías: instrumentales, interpersonales y sistémicas. Las Competencias Instrumentales hacen referencia a habilidades cognitivas, metodológicas, tecnológicas y lingüísticas. Las competencias interpersonales están relacionadas con habilidades sociales de interacción social y cooperación. Por último las Competencias Sistémicas comprenden habilidades que conciernen a sistemas complejos, lo que requiere una combinación de entendimiento, sentido y conocimiento (adquiridas previamente las competencias instrumentales e interpersonales).

Tabla 1. Competencias transversales definidas en el Proyecto Tuning

\begin{tabular}{|c|c|c|}
\hline \multirow{5}{*}{ 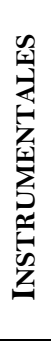 } & Capacidad de análisis y síntesis. & Conocimiento de una segunda lengua. \\
\hline & Capacidad de organizar y planificar. & Habilidades básicas de manejo del ordenador. \\
\hline & Conocimientos generales básicos. & $\begin{array}{l}\text { Habilidades de gestión de la información } \\
\text { (para buscar y analizar información } \\
\text { proveniente de fuentes diversas). }\end{array}$ \\
\hline & Conocimientos básicos de la profesión. & Resolución de problemas. \\
\hline & $\begin{array}{l}\text { Comunicación oral y escrita en la propia } \\
\text { lengua. }\end{array}$ & Toma de decisiones. \\
\hline \multirow{4}{*}{ 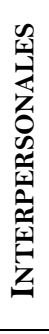 } & Capacidad crítica y autocrítica. & $\begin{array}{l}\text { Capacidad para comunicarse con expertos de } \\
\text { otras áreas. }\end{array}$ \\
\hline & Trabajo en equipo. & $\begin{array}{l}\text { Apreciación de la diversidad y } \\
\text { multiculturalidad. }\end{array}$ \\
\hline & Habilidades interpersonales. & $\begin{array}{l}\text { Habilidad de trabajar en un contexto } \\
\text { internacional. }\end{array}$ \\
\hline & $\begin{array}{l}\text { Capacidad de trabajar en un equipo } \\
\text { interdisciplinar }\end{array}$ & Compromiso ético. \\
\hline \multirow{6}{*}{ 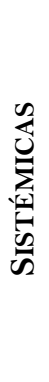 } & $\begin{array}{l}\text { Capacidad de aplicar los conocimientos en } \\
\text { la práctica. }\end{array}$ & $\begin{array}{l}\text { Conocimiento de culturas y costumbres de } \\
\text { otros países. }\end{array}$ \\
\hline & Habilidades de investigación. & Habilidad para trabajar de forma autónoma. \\
\hline & Capacidad de aprender. & Diseño y gestión de proyectos. \\
\hline & $\begin{array}{l}\text { Capacidad para adaptarse a nuevas } \\
\text { situaciones. }\end{array}$ & Iniciativa y espíritu emprendedor. \\
\hline & $\begin{array}{l}\text { Capacidad para generar nuevas ideas } \\
\text { (creatividad). }\end{array}$ & Preocupación por la calidad. \\
\hline & Liderazgo. & Motivación de logro. \\
\hline
\end{tabular}

Fuente: Elaboración propia.

La importancia de esta línea de trabajo para las universidades y para la sociedad es decisiva, tal y como se traduce de los esfuerzos realizados en su elaboración dentro del EEES. En línea con ello, y no necesariamente dentro de nuestras fronteras, se han desarrollado tanto modelos específicos para vincular las competencias de los universitarios con las actividades desarrolladas en entornos específicos de trabajo como las ingenierías (Khairullina et al., 2015), el ámbito médico (Atkinson et al., 2013; Zhao et al., 2015), o el turismo y la hostelería (Jung, 2015); como modelos de corte general que pretenden facilitar la adaptación del universitario desde un nivel superior (Rieckmann, 2012; Shek y Sun, 2012). Este enfoque también ha impulsado el desarrollo de diferentes estrategias de innovación docente tendentes a la mejora de los procesos de aprendizaje y evaluación de los universitarios (Vivel-Búa et al., 2015). 
Sin embargo, para el desarrollo e implantación exitosa de un modelo basado en competencias como éste, con el objetivo de proporcionar a las universidades una apreciación fiable del grado en el que los alumnos manifiestan y desarrollan las mismas, son necesarios dos aspectos fundamentales: de un lado, el desarrollo de un marco común de competencias transversales para los universitarios; y de otro, el desarrollo de instrumentos de medición de dichas competencias.

En lo que se refiere a la primera idea, a nivel nacional, a través de la Agencia Nacional de Evaluación de la Calidad y Acreditación (ANECA) se han creado los Libros Blancos donde se detallan las competencias genéricas y específicas a cada titulación universitaria. Sobre los anteriores trabajos las universidades han tratado de establecer qué competencias eran más demandadas por las organizaciones (p.e., Accenture y Universia, 2007; Gómez y Borrás, 2006; González, 2006). A pesar de ello, como indican López y colaboradores (2007) se requiere un trabajo de análisis y sistematización para llegar a un listado unificado de competencias transversales. En este sentido, los mismos autores, tras realizar un análisis del contenido de las competencias establecidas en diferentes modelos (nacionales e internacionales) proponen un listado integrador de 26 competencias.

Sin embargo, en lo referido a la cuestión de la evaluación, hasta donde llega nuestro conocimiento, es casi inexistente el trabajo desarrollado en nuestro país (a excepción de la propuesta inicial desarrollada por Solanes, Núñez y Rodríguez, 2008). A pesar de que existe ya en el campo una amplia instrumentación estandarizada para la evaluación de competencias mediante cuestionarios, véanse por ejemplo, con alcance internacional los desarrollados de SHL (SHL Group 1993a, 1993b, 1994, 1997, 1999a, 1999b), o el modelo integrado de Blömeke y colaboradores (2013); o en el ámbito nacional y latinoamericano (Instituto de Ingeniería del Conocimiento, 2001; Tea Ediciones, 2008, 2011). A pesar de ello, esta instrumentación no es directamente trasladable al entorno universitario por dos motivos: (a) los modelos sustantivos en los que se basan los cuestionarios responden a modelos de competencias desarrollados en el ámbito organizacional y no contemplan necesariamente las competencias establecidas en los modelos universitarios como Tuning (si bien es cierto que según el modelo pueden compartir algunas de las competencias propuestas); y (b) las evidencias de comportamiento utilizadas como ítems suponen muestras de comportamientos laborales que no suelen ser representativos de las conductas que desarrollan los universitarios.

Por todo ello se requiere aún del desarrollo de instrumentación específica. Esta debe permitir realizar una aproximación estandarizada al grado en el que nuestros estudiantes universitarios manifiestan las diferentes competencias propuestas en los modelos ampliamente aceptados.

\section{Estudio 1}

En línea con lo anterior, el objetivo de nuestro trabajo es desarrollar un cuestionario para la evaluación de competencias transversales en estudiantes universitarios y obtener evidencias iniciales respecto de sus propiedades psicométricas. Para ello en un primer estudio $(N=741)$ se presenta el desarrollo del cuestionario y se analiza su fiabilidad, validez de contenido y estructura dimensional. Posteriormente, en un segundo estudio $(N=46)$ se analiza la validez criterio de las competencias apreciadas por el cuestionario respecto de los resultados académicos. No se proponen hipótesis específicas, pero se 
espera que las escalas del cuestionario sean fiables (Alfa de Cronbach superior a 0,70), que tengan una alta validez de contenido (Índice de Congruencia superior a 0,80 ) y que muestren una estructura latente congruente con el modelo Tuning. Igualmente, se espera que las competencias apreciadas por el cuestionario estén correlacionadas con el rendimiento académico y que proporcionen un incremento de varianza explicada en éste sobre el proporcionado por la inteligencia y la personalidad.

\subsection{Método}

\section{Participantes}

741 participantes de último curso de diferentes titulaciones de una universidad pública de la Comunidad de Madrid (56\% de ellos varones; media de edad 22 años). Por ramas de conocimiento la distribución de los participantes fue la siguiente: ciencias sociales y jurídicas $(51 \%)$, ciencias $(27 \%)$, ingeniería y arquitectura (18\%), y ciencias de la salud $(5 \%)$. Todos los participantes cumplimentaron el cuestionario en el marco de un programa especial para el desarrollo de competencias que se estaba llevando a cabo en la universidad. Su participación en el programa era voluntaria y no recibieron compensación económica. Todos los participantes firmaron un documento de consentimiento informado aceptando que sus datos fueran tratados estadísticamente con el objetivo de analizar las medidas obtenidas.

\section{Instrumento}

Cuestionario de Evaluación de Competencias Transversales en Universitarios. El cuestionario consta de 185 elementos en un formato de evidencias de comportamiento que se responde en una escala de frecuencia de cuatro puntos (1. Nunca-Casi Nunca; 2. Pocas Veces; 3. A menudo; 4. Siempre-Casi Siempre). Se aprecian las 26 competencias para universitarios derivadas del Proyecto Tuning por López y colaboradores (2007) (ver Apéndice I). La presentación de los ítems en formato de evidencia de comportamiento responde al criterio de utilización de escalas tipo BOs (Behovioral Observation Scales) que para la evaluación de competencias han demostrado ser fiables y válidas (Latham y Wexley, 1981; Murphy, Martin y García, 1982; Wiersma y Latham, 1986). El desarrollo de las escalas fue realizado según los estándares al uso.

Siguiendo las instrucciones proporcionadas por nuestro equipo de investigación acerca de cómo redactar elementos de medida, tres expertos desarrollaron ítems para cada uno de los veintiséis objetivos de evaluación propuestos. Se escogieron expertos en el desarrollo de evidencias de comportamiento para competencias en el ámbito organizacional (dos expertos) y en el diseño de programas de desarrollo de habilidades en universitarios para favorecer su inserción laboral (un experto) y cada uno de ellos trabajó de manera independiente. Fruto del trabajo del equipo de expertos se desarrollaron 390 evidencias de comportamiento (de media, 15 evidencias por competencia). Nuestro equipo analizó todas las evidencias desarrolladas con el objetivo de eliminar redundancias y elementos mal diseñados. Fruto de este trabajo se retuvieron 228 elementos. Los 228 elementos se pusieron a prueba en diferentes aplicaciones controladas y tras el análisis de sus propiedades se conservaron únicamente los 185 elementos que conforman el cuestionario. No todas las escalas son apreciadas con el mismo número de elementos sino que atendiendo al dominio de contenidos a evaluar y a las características métricas de los ítems las escalas cuentan con 5, 7 o 10 elementos. En la tabla 2 puede encontrarse (señalado entre paréntesis) el número de elementos que 
forman cada escala. Finalmente el cuestionario se construyó presentando los elementos siguiendo un orden establecido de forma aleatoria. El cuestionario está diseñado para ser administrado en formato informatizado a través de una página Web y no fue administrado en ningún caso a través de formato de lápiz y papel. En el Apéndice I adicionalmente al contenido de evaluación para cada competencia se muestran dos ejemplos de los ítems utilizados (el cuestionario está disponible bajo petición en la dirección del primer autor).

\section{Procedimiento}

El cuestionario fue administrado a través de una página web. Los participantes lo cumplimentaron en un conjunto de aulas informáticas de la Universidad controladas por nuestro equipo de investigación. En cuanto al análisis de datos, para las escalas e ítems se obtuvieron las medias, desviaciones típicas y el índice de discriminación de cada ítem (correlación ítem-total corregida con la escala de referencia).

La fiabilidad de las escalas se apreció a partir del coeficiente Alfa de Cronbach. A pesar de que con escalas de respuesta de cuatro o menos categorías (como es nuestro caso) se produce una infra-estimación de su verdadero valor (Lozano, García-Cueto y Muñiz, 2008; Weng, 2004), y otros métodos como el Alfa Ordinal o el coeficiente Theta de Armor han sido propuestos (Elosua y Zumbo, 2008), la disponibilidad de estos en los paquetes estadísticos habituales es escasa y su utilización está muy poco extendida.

El análisis de la validez de contenido de los ítems se realizó obteniendo el índice de congruencia de Rovinelli y Hambleton (1977) para cada ítem. Cinco jueces expertos ajenos al equipo de investigación y diferentes de los expertos que desarrollaron el cuestionario, indicaron la adecuación de cada ítem (no adecuado/sí adecuado) para la evaluación de cada uno de los objetivos de evaluación y sobre sus respuestas se calculó el mencionado índice. Los jueces desarrollaron su tarea de validación de manera paralela a la administración del cuestionario a los participantes.

Finalmente el análisis de la estructura latente del cuestionario se realizó a través de análisis factorial exploratorio.

\subsection{Resultados}

En la tabla 2 se muestran los estadísticos descriptivos básicos de las escalas que componen el cuestionario. Las medias obtenidas en las escalas están por encima de los valores medios teóricos (12,5 para las escalas con 5 ítems; 17,5 para las escalas con 7 ítems; 25 para las escalas con 10 ítems). Así, las escalas que obtienen una mayor puntuación media son C25: Responsabilidad Laboral, C18: Habilidades Informáticas Básicas, C21: Asertividad, C23: Conocimiento y Valoración de la Diversidad, C13: Seguridad en sí mismo, C10: Trabajo en Equipo, Co8: Orientación al Cliente y C11: Comunicación; mientras que las escalas en las que se obtienen puntuaciones más bajas son C24: Responsabilidad Social, Co5: Innovación, C12: Iniciativa, C06: Liderazgo, C03: Capacidad de Análisis y Síntesis, y C16: Negociación-Persuasión.

Teniendo en cuenta el promedio de los índices de congruencia obtenidos para los distintos ítems que conforman las escalas, observamos que 14 de las 26 escalas propuestas obtienen un promedio de índices de congruencia superiores a 0,90 (por ejemplo, Capacidad de Aprendizaje, Autocontrol, Conocimiento y Valoración de la Diversidad). Otras 6 escalas obtienen un promedio de índices de congruencia superior a 
0,80 (por ejemplo, Capacidad de Análisis, Negociación-Persuasión). Hay tres escalas con valores superiores a 0,70 pero que no llegan al 0,80: Orientación a Resultados (0,79), Iniciativa $(0,76)$ y Seguridad en sí Mismo $(0,77)$. Sin embargo aparecen tres escalas en las que los índices de congruencia son bajos. Se trata de las escalas de Asertividad $(0,41)$ y Capacidad de Crítica y Autocrítica $(0,65)$ que ya mostraron los índices de fiabilidad más bajos, y la escala Habilidad para Trabajar de Forma Autónoma $(0,42)$. La valoración de la calidad de los índices obtenidos puede realizarse siguiendo el criterio propuesto por Osterlind (1989). Según el autor los índices de congruencia superiores a 0,50 han de ser considerados como aceptables. En esta línea, todos los elementos del cuestionario, excepto dos ítems de la escala Habilidad para Trabajar de Forma Autónoma y un ítem de la escala de Asertividad, cumplen con el estándar fijado.

Adicionalmente, el promedio del coeficiente de discriminación de los ítems estimado para cada escala también es mostrado. Como se ve es superior a 0,40 excepto para las escalas de Asertividad, Capacidad de Crítica y Autocrítica y Responsabilidad Laboral. El mayor promedio se encuentra, como era de esperar, para las escalas con un mayor indicador de consistencia. Además es necesario señalar que todos los ítems superaron el criterio de retención propuesto por Schmeiser y Welch (2006) situado en 0,20 a excepción de un elemento de la escala C8: Orientación al Cliente y otro de la escala C22: Capacidad de Crítica y Autocrítica.

Al observar el coeficiente Alfa estimado para cada una de las escalas se aprecia cómo para todas ellas se obtiene un valor satisfactorio $(>0,70)$ a excepción de las escalas de Asertividad $(0,67)$ y Capacidad de Crítica y Autocrítica $(0,59)$. La escala que mayor fiabilidad obtiene es la de Innovación $(0,86)$ junto con las de Liderazgo $(0,81)$ y Comunicación $(0,81)$.

Respecto de las correlaciones entre las variables, puede apreciarse (ver tabla 3) cómo todas ellas son significativas $(\mathrm{p}<0,01)$ a excepción de las que mantiene Responsabilidad Social con Capacidad de Análisis y Síntesis $\left(\mathrm{r}_{\mathrm{xy}}=0,09 ; \mathrm{p}>0,05\right)$ y con Habilidades Informáticas Básicas $\left(r_{\mathrm{xy}}=0,04\right.$; no significativa); y las que se encuentran entre Conocimiento y Valoración de la Diversidad con Habilidades Informáticas Básicas ( $\mathrm{r}_{\mathrm{xy}}=$ $0,09 ; \mathrm{p}>0,05)$ y con Interés por la Calidad ( $\mathrm{r}_{\mathrm{xy}}=0,08$; no significativa).

Adicionalmente, en la tabla 4 se presenta la asociación de escalas hallada al factorizar las puntuaciones en las escalas utilizando el método de factorización de los ejes principales. Atendiendo a la correlación existente entre las escalas se optó por la rotación oblicua oblimín con Kaiser. Tanto el estadístico de Kaiser-Meyer-Olkin $(0,956)$ como la prueba de esfericidad de Barlett (Chi-cuadrado aproximado $=6421,216$; sig $=0,000$ ) indicaron la adecuación de la matriz de correlaciones para proceder a su factorización. Además, la prueba de bondad de ajuste de la extracción realizada, también resultó ser significativa (Chi-cuadrado 473,198 con 227 grados de libertad es significativo a nivel $\mathrm{p}<0,000$ ).

$\mathrm{Al}$ segundo factor extraído (6,7 \% de la varianza total explicada) parecen asociarse las competencias de Planificación y Organización, Interés por la Calidad, Habilidad para Trabajar de Forma Autónoma, Responsabilidad Laboral y Orientación a Resultados. Podría etiquetarse como competencias para la Acción. Al tercer factor extraído (3,4\% de la varianza total explicada) se asocian las competencias de Orientación al Cliente, Trabajo en Equipo, Asertividad, Comunicación, Liderazgo y Capacidad de Crítica y Autocrítica. Podría ser etiquetado como competencias para la Interacción. Finalmente, el cuarto factor extraído (2,6\% de la varianza total explicada) recoge las competencias 
Responsabilidad Social, Conocimiento y Valoración de la Diversidad y Habilidades de Búsqueda y Gestión de la Información. Podría ser etiquetado como competencias para el Compromiso Social.

Tabla 2. Estadísticos descriptivos, índices de congruencia, discriminación y coeficiente Alfa de las escalas

\begin{tabular}{|c|c|c|c|c|c|}
\hline $\operatorname{ESCALA}(\boldsymbol{K})$ & $\mathbf{M}$ & DT & CONG. (MIN-MAX) & DISCR. (MIN-MAX) & ALFA \\
\hline $\operatorname{Co1}(5)$ & 15,13 & 2,64 & $0,99(, 97-1)$ & $0,58(, 42-, 72)$ & 0,79 \\
\hline $\mathrm{Co} 2(5)$ & 15,93 & 2,22 & $0,93(, 74-1)$ & $0,46(, 41-, 54)$ & 0,7 \\
\hline $\operatorname{Co3}(5)$ & 16,17 & 2,26 & $0,89(, 87-1)$ & $0,5(, 42-, 56)$ & 0,74 \\
\hline $\operatorname{Co4}(7)$ & 21,42 & 3,5 & $0,95(, 61-1)$ & $0,52(, 34-, 61)$ & 0,79 \\
\hline $\operatorname{Co5}(5)$ & 13,63 & 3,12 & $0,93(, 82-1)$ & $0,68(, 60-, 75)$ & 0,86 \\
\hline $\operatorname{Co6}(10)$ & 30,07 & 4,92 & $0,82(, 51-1)$ & $0,49(, 42-, 61)$ & 0,81 \\
\hline $\operatorname{Co7}(10)$ & 31,74 & 4,64 & $0,92(, 7-1)$ & $0,46(, 36-, 56)$ & 0,79 \\
\hline $\operatorname{Cos}(10)$ & 32,58 & 3,82 & $0,91(, 6-1)$ & $0,41(, 15-, 50)$ & 0,74 \\
\hline $\operatorname{Co9}(7)$ & 21,51 & 3,24 & $0,79(, 6-1)$ & $0,46(, 38-, 53)$ & 0,74 \\
\hline $\mathrm{C} 10(10)$ & 32,64 & 4,1 & $0,93(, 6-1)$ & $0,44(, 31-, 62)$ & 0,77 \\
\hline C11 (10) & 32,58 & 4,38 & $0,95(, 6-1)$ & $0,5(, 36-, 67)$ & 0,81 \\
\hline $\mathrm{C} 12(7)$ & 19,95 & 3,59 & $0,76(, 6-, 9)$ & $0,48(, 36-, 57)$ & 0,76 \\
\hline C13 (7) & 22,89 & 2,9 & $0,77(, 6-1)$ & $0,41(, 25-, 47)$ & 0,7 \\
\hline $\mathrm{C} 14(5)$ & 15,32 & 2,69 & $0,96(, 8-1)$ & $0,51(, 48-, 51)$ & 0,74 \\
\hline C15 (7) & 22,54 & 3,04 & $0,92(, 5-1)$ & $0,44(, 26-, 50)$ & 0,73 \\
\hline $\mathrm{C} 16(7)$ & 21,19 & 3,31 & $0,86(, 6-1)$ & $0,48(, 43-, 54)$ & 0,76 \\
\hline $\mathrm{C} 17$ (5) & 16,02 & 2,54 & $0,92(, 8-1)$ & $0,52(, 48-, 55)$ & 0,75 \\
\hline C18 (5) & 16,7 & 2,71 & $0,95(, 9-1)$ & $0,47(, 37-, 53)$ & 0,7 \\
\hline C19 (7) & 21,98 & 3,09 & $0,84 \cdot(, 8-, 9)$ & $0,49(, 40-, 58)$ & 0,77 \\
\hline $\mathrm{C} 2 \mathrm{O}(5)$ & 15,29 & 2,31 & $0,98(, 9-1)$ & $0,5(, 37-, 57)$ & 0,73 \\
\hline $\mathrm{C} 21(10)$ & 33,19 & 3,59 & $0,41(, 3-, 9)$ & $0,34(, 25-, 43)$ & 0,67 \\
\hline $\mathrm{C}_{22}(7)$ & 21,44 & 2,95 & $0,65(, 5-, 9)$ & $0,32(, 19-, 47)$ & 0,59 \\
\hline $\mathrm{C}_{23}(7)$ & 22,96 & 3,78 & $0,97(, 9-1)$ & $0,54(, 28-, 62)$ & 0,8 \\
\hline $\mathrm{C} 24(5)$ & 13,34 & 3,18 & $0,89(, 5-, 9)$ & 0,53 (,39-,65) & 0,76 \\
\hline $\mathrm{C} 25(10)$ & 34,06 & 3,58 & $0,83(, 5-, 9)$ & $0,37(, 25-, 48)$ & 0,71 \\
\hline C26 (7) & 22,14 & 3,13 & $0,42(, 3-, 8)$ & $0,44(, 34-, 54)$ & 0,73 \\
\hline
\end{tabular}

Nota: C01: Capacidad de Aprendizaje; C02: Utilización de Conocimientos; Co3: Capacidad de Análisis y Síntesis; Co4: Habilidades de Búsqueda y Gestión de Información; Co5: Innovación; C06: Liderazgo; C07: Interés por la Calidad; Co8: Orientación al Cliente; Co9: Orientación a Resultados; C10: Trabajo en Equipo; C11: Comunicación; C12: Iniciativa; C13: Seguridad en sí mismo; C14: Autocontrol; C15: Flexibilidad-Gestión del Cambio; C16: Negociación-Persuasión; C17: Planificación y Organización; C18: Habilidades Informáticas Básicas; C19: Resolución de Problemas; C20: Toma de Decisiones; C21: Asertividad; C22: Capacidad de crítica y autocrítica; C23: Conocimiento y Valoración de la Diversidad; C24: Responsabilidad Social; C25: Responsabilidad Laboral; C26: Habilidad para trabajar de forma Autónoma; Escala $(K)$ : escala (entre paréntesis número de ítems que la componen); Cong. (min-max): promedio de los índices de congruencia de los ítems de la escala (entre paréntesis el mínimo y el máximo obtenido); Discr. (min-max): promedio de las correlaciones ítem-total corregidas de los ítems de la escala (entre paréntesis el mínimo y el máximo obtenido).

Fuente: Elaboración propia. 
Tabla 3. Estadísticos descriptivos, índices de congruencia, discriminación, alfa e interrelaciones entre escalas

\begin{tabular}{|c|c|c|c|c|c|c|c|c|c|c|c|c|c|c|c|c|c|c|c|c|c|c|c|c|c|c|}
\hline & Escala & 2 & 3 & 4 & 5 & 6 & 7 & 8 & 9 & 10 & 11 & 12 & 13 & 14 & 15 & 16 & 17 & 18 & 19 & 20 & 21 & 22 & 23 & 24 & 25 & 26 \\
\hline 1 & $\mathrm{Co} 1(5)$ &, 52 &, 52 & ,36 &, 42 & ,46 & ,29 & ,44 & ,49 & ,28 &, 42 &, 53 &, 43 &, 47 &, 50 & ,46 & ,30 & ,35 &, 54 &, 47 & ,32 & ,29 &, 19 & $09^{1}$ &, 21 &, 45 \\
\hline 2 & $\mathrm{Co} 2(5)$ & & ,60 & ,56 &, 55 &, 54 & ,43 & ,63 &, 61 & ,43 &, 58 & ,61 &, 57 &, 51 &, 58 & ,62 &, 47 & ,26 & ,66 &, 62 & , 46 &, 45 &, 27 & ,22 &, 34 &, 58 \\
\hline 3 & $\operatorname{Co3}(5)$ & & & ,49 & ,53 & ,56 & ,49 &, 58 & ,63 &, 45 &, 72 &, 57 &, 52 &, 52 &, 58 &, 64 & ,61 & ,30 &, 73 &, 74 &, 51 &, 45 & ,26 &, 27 &, 38 & ,62 \\
\hline 4 & $\mathrm{CO} 4(7)$ & & & & ,46 & ,45 &, 50 &, 58 & ,62 & ,43 & ,46 &, 57 &, 51 &, 40 &, 51 &, 55 &, 44 &, 27 &, 54 &, 50 & ,43 &, 47 & ,34 &, 42 & ,36 &, 53 \\
\hline 5 & $\operatorname{Co5}(5)$ & & & & &, 57 & ,29 & ,49 & ,59 & ,38 & ,46 & ,68 &, 47 &, 47 &, 51 &, 58 & ,33 &, 24 & ,60 &, 52 & ,39 &, 47 &, 28 & ,29 &, 13 &, 44 \\
\hline 6 & $\operatorname{Co6}(10)$ & & & & & &, 42 & ,63 & ,63 & ,68 &, 58 &, 72 & ,53 &, 55 & ,64 & ,66 &, 44 & ,22 &, 59 &, 56 &, 56 & ,48 & ,33 &, 27 &, 38 &, 52 \\
\hline 7 & $\mathrm{Co} 7(10)$ & & & & & & & ,59 & ,53 &, 31 &, 45 &, 44 &, 42 & ,36 & ,43 &, 37 & ,65 & ,32 &, 49 &, 53 & ,34 & ,31 & $08^{2}$ &, 14 &, 52 & ,68 \\
\hline 8 & $\operatorname{Cos}(10)$ & & & & & & & & ,68 & ,64 & ,66 & ,62 &, 55 & ,49 & ,67 & ,66 & ,61 & ,32 & ,62 &, 64 & ,61 &, 51 & ,32 & ,29 &, 55 & ,67 \\
\hline 9 & $\operatorname{Co9}(7)$ & & & & & & & & &, 50 &, 58 &, 71 &, 57 & ,53 &, 59 &, 67 &, 61 &, 31 & ,67 &, 67 & 49 &, 51 &, 25 &, 34 &, 43 & ,64 \\
\hline 10 & $\mathrm{C} 10(10)$ & & & & & & & & & &, 52 &, 52 & 49 & ,44 &, 67 &, 55 &, 37 &, 17 & ,46 &, 40 & ,63 &, 55 &, 44 & ,33 &, 37 &, 37 \\
\hline 11 & C11 (10) & & & & & & & & & & &, 56 &, 56 &, 50 & ,59 &, 71 &, 51 &, 26 & ,62 & ,64 & ,61 & ,48 & ,32 & ,23 & ,39 &, 55 \\
\hline 12 & $\mathrm{C} 12(7)$ & & & & & & & & & & & &, 59 & ,60 &, 64 &, 70 & ,46 &, 32 & ,68 &, 61 &, 50 &, 52 &, 31 & ,29 &, 32 &, 58 \\
\hline 13 & $\mathrm{C} 13(7)$ & & & & & & & & & & & & & ,59 & ,62 &, 60 & ,43 & ,24 &, 56 &, 54 &, 54 &, 52 &, 27 & ,23 &, 35 &, 54 \\
\hline 14 & $\mathrm{C} 14(5)$ & & & & & & & & & & & & & & ,62 &, 61 & ,38 &, 27 &, 58 &, 56 &, 50 & ,46 &, 24 &, 20 &, 30 &, 52 \\
\hline 15 & $\mathrm{C} 15$ (7) & & & & & & & & & & & & & & &, 61 &, 47 & ,30 & ,60 &, 57 & ,61 &, 55 & ,33 & ,29 &, 41 &, 54 \\
\hline 16 & $\mathrm{C} 16(7)$ & & & & & & & & & & & & & & & &, 48 & ,26 & ,67 & ,62 &, 58 &, 50 & ,30 & ,39 &, 31 &, 56 \\
\hline 17 & $\mathrm{C} 17(5)$ & & & & & & & & & & & & & & & & & ,23 &, 58 &, 61 &, 40 & ,33 &, $10^{1}$ &, 22 &, 48 &, 77 \\
\hline 18 & $\mathrm{C} 18(5)$ & & & & & & & & & & & & & & & & & & ,26 &, 25 &, 15 &, 17 & $09^{1}$ &, $04^{2}$ &, 26 & ,33 \\
\hline 19 & C19 (7) & & & & & & & & & & & & & & & & & & &, 75 & 49 & ,49 &, 19 & ,20 & ,36 &, 64 \\
\hline 20 & $\mathrm{C} 20(5)$ & & & & & & & & & & & & & & & & & & & &, 47 &, 47 &, 15 & ,23 &, 36 & ,65 \\
\hline 21 & $\mathrm{C}_{21}(10)$ & & & & & & & & & & & & & & & & & & & & & ,53 & ,38 & ,25 &, 40 & ,43 \\
\hline 22 & $\mathrm{C} 22(7)$ & & & & & & & & & & & & & & & & & & & & & &, 40 & ,36 &, 31 &, 34 \\
\hline 23 & $\mathrm{C} 23$ (7) & & & & & & & & & & & & & & & & & & & & & & & ,45 &, 21 &, 11 \\
\hline 24 & $\mathrm{C} 24 .(5)$ & & & & & & & & & & & & & & & & & & & & & & & &, 25 &, 17 \\
\hline 25 & $\mathrm{C} 25(10)$ & & & & & & & & & & & & & & & & & & & & & & & & &, 48 \\
\hline
\end{tabular}

Nota: Todas las correlaciones significativas al nivel 0,01 (bilateral) excepto 1 Sig. 0,05 (bilateral) y 2 No Sig.

Fuente: Elaboración propia. 
Las correlaciones entre los factores extraídos son positivas entre los factores 1 , 3 y 4 y negativas en todas las correlaciones con el factor 2 (a pesar de ello se indica una relación directa entre lo apreciado por el factor 2 y el resto de factores ya que las saturaciones factoriales en dicho factor son negativas, como se puede apreciar en la tabla 4). La cuantía de estas correlaciones $\left(r_{1,2}=-0,577 ; r_{1,3}=0,535 ; r_{1,4}=0,408 ; r_{2,3}=-0,399 ; r_{2,4}=-\right.$ 0,$\left.245 ; r_{3,4}=0,446\right)$ muestra una alta relación entre los factores extraídos. Por otro lado es necesario considerar que existen algunas de las competencias que muestran saturaciones pobres con su factor principal. Es el caso de las Habilidades Informáticas Básicas, de la Orientación a Resultados, de la Orientación al Cliente, y de la Capacidad de Crítica y Autocrítica.

\section{Estudio 2}

El objetivo de este segundo estudio es examinar la capacidad de las competencias apreciadas con el cuestionario para predecir el rendimiento académico y, adicionalmente, examinar la validez incremental (respecto de la inteligencia y la personalidad) tomando el mismo criterio de rendimiento académico.

\subsection{Método}

\section{Participantes}

Participaron 46 alumnos de una titulación del ámbito de las Ciencias Sociales y Jurídicas de la misma Universidad en la que se desarrolló el estudio 1. La edad media fue 23,3 años y la distribución por sexos fue de un $75 \%$ mujeres. Los participantes realizaron la investigación de manera voluntaria y firmaron el documento de consentimiento informado antes de comenzar, donde se les informaba acerca de la utilización de los datos recogidos con fines estadísticos. Al finalizar la recogida de datos los alumnos fueron informados de los objetivos del estudio, así como de los resultados que habían obtenido en las diferentes pruebas.

\section{Medidas}

- Competencias transversales. Se utilizó el cuestionario desarrollado y analizado en el primer estudio de este trabajo, obteniéndose una puntuación para cada una de las 26 competencias apreciadas.

- Inteligencia General. Se utilizó el Test de Factor G, propiedad del Instituto de Ingeniería del Conocimiento (IIC) y que consta de 40 ítems. El formato de los ítems es el de series lógicas de figuras que deben completarse. Se presenta una serie de 3 figuras a la que sigue una cuarta que no se especifica. La respuesta debe elegirse de entre 4 opciones de las que solo una es correcta. En los estudios previos (Aguado y Lucía, s.f.) el test obtuvo unas puntuaciones aceptables en los análisis de fiabilidad (rango de puntuaciones Alfa de Cronbach entre 0,65 y 0,74$)$ y de validez (correlación significativa $r_{x y}=0,44 ; p<$ 0,01 con el test de Matrices Progresivas de Raven).

- Personalidad. La evaluación de las variables de personalidad se basó en la teoría de los Big Five (Costa y McCrae, 1985, 1992). Se empleó el cuestionario BFCP Internet en su versión reducida de 60 ítems (Aguado et al., 2008) y se 
obtuvieron las puntuaciones en los cinco grandes factores: ajuste emocional, extraversión, cordialidad, responsabilidad y apertura a la experiencia.

Tabla 4. Solución factorial rotada (matriz de configuración) sobre la puntuación en las 26 escalas

\begin{tabular}{|c|c|c|c|c|}
\hline & F1 & F2 & F3 & F4 \\
\hline Innovación & 0,881 & & & \\
\hline Resolución de Problemas & 0,693 & & & \\
\hline Capacidad de Aprendizaje & 0,672 & & & \\
\hline Iniciativa & 0,669 & & & \\
\hline Autocontrol & 0,643 & & & \\
\hline Capacidad de Análisis y Síntesis & 0,592 & $-0,348$ & & \\
\hline Toma de Decisiones & 0,543 & $-0,423$ & & \\
\hline Negociación-Persuasión & 0,541 & & & \\
\hline Utilización de Conocimientos & 0,523 & & & \\
\hline Seguridad en sí mismo & 0,474 & & & \\
\hline Flexibilidad-Gestión del Cambio & 0,444 & & 0,434 & \\
\hline Habilidades Informáticas Básicas & 0,337 & & & \\
\hline Planificación y Organización & & $-0,830$ & & \\
\hline Interés por la Calidad & & $-0,798$ & & \\
\hline H. Trabajar Autónoma & & $-0,733$ & & \\
\hline Responsabilidad Laboral & & $-0,649$ & & \\
\hline Orientación a Resultados & 0,394 & $-0,398$ & & \\
\hline Orientación al Cliente & & $-0,384$ & 0,374 & \\
\hline Trabajo en Equipo & & & 0,769 & \\
\hline Asertividad & & & 0,678 & \\
\hline Comunicación & 0,334 & & 0,493 & \\
\hline Liderazgo & 0,381 & & 0,466 & \\
\hline Capacidad de crítica y autocrítica & 0,333 & & 0,339 & \\
\hline Responsabilidad Social & & & & 0,623 \\
\hline Habilidades de B. y G. Información & & $-0,330$ & & 0,548 \\
\hline Cto. y Val . Diversidad & & & 0,332 & 0,458 \\
\hline
\end{tabular}

Nota: Método de extracción: ejes principales; Método de rotación: Oblimín con Kaiser; Convergencia: 15 iteraciones.

Fuente: Elaboración propia.

- Rendimiento académico. Se emplearon tres medidas de Rendimiento Académico. Por un lado se contempló la puntuación obtenida por los alumnos en su evaluación continua. Esta puntuación se obtiene de la valoración que el profesor realiza de las prácticas y ejercicios realizados en clase. Por otro lado se contempló el resultado obtenido por los alumnos en el examen de conocimientos de la asignatura. Finalmente se consideró la puntuación final en la asignatura obtenida por cada alumno. Esta puntuación final tiene en cuenta las dos puntuaciones anteriores pero es corregida por el profesor a su criterio introduciendo su apreciación subjetiva del rendimiento del alumno. Tanto la valoración continua, como el examen y la calificación final se puntuaron en una escala de 1-10. 


\section{Procedimiento}

La recogida de datos se efectuó dentro de las prácticas de la asignatura. En la práctica los alumnos examinaron diferentes métodos de evaluación on-line para la selección de personal. Después y como parte de la investigación, cumplimentaron las diferentes medidas. La aplicación se realizó de forma concurrente en un aula de informática en un entorno supervisado. En cuanto al análisis de datos realizado, para el estudio de la validez de las competencias transversales en la explicación de las calificaciones académicas se llevaron a cabo diferentes análisis de regresión tomando como variable dependiente cada una de las variables utilizadas en el estudio como indicadores del rendimiento académico (evaluación continua, evaluación examen y evaluación final).

\subsection{Resultados}

Los estadísticos descriptivos básicos que se han obtenido en las diferentes escalas aparecen reflejados en la tabla 5. Se muestra cómo los valores medios en las escalas correspondientes a las competencias evaluadas se aproximan a los valores teóricos para escalas de 5, 7 o 10 ítems que ya se comentaron en el primer estudio. Las escalas con mayor puntuación media son Responsabilidad Laboral, Asertividad, Comunicación y Orientación al Cliente, mientras que las escalas para las que se obtuvo un menor promedio fueron Responsabilidad Social, Innovación, Capacidad de Aprendizaje y Toma de Decisiones. Por su parte, se explicitan la media y la desviación típica correspondientes a las variables criterio Evaluación Continua $(\mathrm{M}=7,24, \mathrm{DT}=0,84)$, Evaluación Examen $(\mathrm{M}=7,11, \mathrm{DT}=1,77)$ y Evaluación Final $(\mathrm{M}=14,38, \mathrm{DT}=2,06)$, así como del Factor $\mathrm{G}(\mathrm{M}=27,57, \mathrm{DT}=4,15)$ y de las variables de personalidad en términos de Big Five (Ajuste emocional $\mathrm{M}=58,17, \mathrm{DT}=9,55$; Extraversión $\mathrm{M}=53,02, \mathrm{DT}=6,96$; Cordialidad $\mathrm{M}=55,78, \mathrm{DT}=5,72$; Responsabilidad $\mathrm{M}=55,37$, DT $=7,82$; Apertura a la experiencia $\mathrm{M}=53,22, \mathrm{DT}=8,13$ ).

En lo que respecta a la fiabilidad de las escalas, en la diagonal de la tabla 5 se muestra los valores del Alfa de Cronbach para cada una de ellas. Puede verse que se obtienen valores ligeramente inferiores a los obtenidos en el estudio anterior en algunas de las variables, probablemente debido al tamaño de la muestra.

En cuanto a las correlaciones entre las variables de personalidad, todas ellas son significativas a excepción de Ajuste Emocional con Responsabilidad; Extraversión con Apertura a la Experiencia, Cordialidad con Responsabilidad; y Responsabilidad con Apertura a la experiencia.

En lo que concierne a las intercorrelaciones entre las 26 competencias genéricas, todas ellas muestran una gran relación, con correlaciones significativas entre la gran mayoría de ellas. Aquellas que muestran una menor relación con el resto son Responsabilidad Laboral, Habilidades Informáticas Básicas y Conocimiento y Valoración de la Diversidad, en orden de menor a mayor número de correlaciones significativas con otras competencias (respectivamente 3 correlaciones significativas, 1 de ellas a nivel $\mathrm{p}<0,01 ; 7$ correlaciones significativas, 1 a nivel $\mathrm{p}<0,01$; y 10 correlaciones significativas, siendo 4 de ellas a nivel $\mathrm{p}<0,01)$.

Las competencias de nuestro modelo no muestran ninguna correlación significativa con el Factor G. Sin embargo, la variable de personalidad Ajuste Emocional sí muestra una correlación significativa $\left(r_{x y}=0,29 ; p<0,01\right)$ con Factor $\mathrm{G}$. 
Por su parte, las correlaciones entre las competencias transversales y las escalas de Big Five muestran una gran relación, con 13 competencias relacionadas significativamente con Ajuste Emocional ( 5 de ellas con $\mathrm{p}<0,01), 18$ con Extraversión (11 a nivel $\mathrm{p}<0,01$ ), 19 con Responsabilidad (6 de ellas a nivel $\mathrm{p}<0,01)$ y 18 con Apertura a la Experiencia (5 a nivel $p<0,01)$. Finalmente, la escala de Cordialidad correlaciona significativamente con 11 competencias (siendo 8 de ellas a nivel $p<0,01$ ).

Finalmente, en la tabla 5 se muestran las intercorrelaciones entre las diferentes escalas, incluidas las variables criterio: Evaluación Continua, Evaluación Examen y Evaluación final. Debe señalarse que hay cuatro competencias genéricas que correlacionan de forma significativa con Evaluación Final. Estas son Capacidad de Aprendizaje $\left(\mathrm{r}_{\mathrm{xy}}=0,38 ; \mathrm{p}<\right.$ 0,05), Interés por la Calidad $\left(\mathrm{r}_{\mathrm{xy}}=0,36 ; \mathrm{p}<0.01\right)$, Seguridad en sí Mismo $\left(\mathrm{r}_{\mathrm{xy}}=0,37 ; \mathrm{p}<\right.$ $0,01)$ y Habilidad para Trabajar de Forma Autónoma $\left(\mathrm{r}_{\mathrm{xy}}=0,33 ; \mathrm{p}<0,01\right)$. Así mismo, cuatro son las competencias que correlacionan significativamente con Evaluación Continua, siendo estas Interés por la Calidad $\left(\mathrm{r}_{\mathrm{xy}}=0,39 ; \mathrm{p}<0,05\right)$, Orientación al Cliente $\left(\mathrm{r}_{\mathrm{xy}}=0,31 ; \mathrm{p}<0,01\right)$, Seguridad en sí Mismo $\left(\mathrm{r}_{\mathrm{xy}}=0,32 ; \mathrm{p}<0,01\right)$ y Habilidad para Trabajar de Forma Autónoma $\left(\mathrm{r}_{\mathrm{xy}}=0,33 ; \mathrm{p}<0,01\right)$. Por último, la variable Evaluación Examen muestra correlación con la competencia Capacidad de Aprendizaje $\left(r_{x y}=0,43\right.$; $\mathrm{p}<0,01)$.

Con el objetivo de establecer el grado en el que las diferentes variables estudiadas permiten predecir los criterios académicos utilizados realizamos diferentes análisis de regresión tomando como variable dependiente la Evaluación Continua, la Evaluación Examen y la Evaluación Final.

Al tomar como variable dependiente la Evaluación Continua introdujimos por pasos en el modelo de regresión las competencias que habían mostrado una correlación significativa con ella: C07: Interés por la Calidad; C08: Orientación al Cliente; C13: Seguridad en sí Mismo; y C26: Habilidad para Trabajar de Forma Autónoma. Sólo la primera de las competencias (C07: Interés por la Calidad) fue introducida en el modelo que resultó significativo $\left(\mathrm{F}_{1,41}=7,493\right.$; Sig. 0,009$)$ con un valor de $\mathrm{R}=0,393 \mathrm{y}$ un incremento de varianza explicada $\Lambda \mathrm{R}^{2}=0,155$.

Teniendo en cuenta como variable dependiente la Evaluación Examen, se introdujo en el modelo únicamente la competencia Co1: Capacidad de Aprendizaje, el modelo resultante fue significativo $\left(F_{1,41}=9,343\right.$; Sig. 0,004) con $R=0,431$ y $\wedge R^{2}=0,186$.

Tomando como variable dependiente la Evaluación Final se introdujeron por pasos las competencias C01: Capacidad de Aprendizaje; Co7: Interés por la Calidad; C13: Seguridad en sí Mismo; y C26: Habilidad para Trabajar de Forma Autónoma. El modelo resultante introdujo únicamente la Co1: Capacidad de Aprendizaje, con un $\mathrm{R}=0,383$, $\Lambda \mathrm{R}^{2}=0,127$ significativo $\left(\mathrm{F}_{1,43}=7,393\right.$; Sig. 0,009). 
Tabla 5. Intercorrelaciones entre Test de Factor G, BFCP Internet y CCT en Universitarios

\begin{tabular}{|c|c|c|c|c|c|c|c|c|c|c|c|c|c|c|c|c|c|c|c|c|c|c|c|c|c|c|c|c|c|c|c|c|c|c|c|c|c|}
\hline & & $\mathrm{M}$ & DT & B & $\bar{c}$ & $\bar{D}$ & $\bar{E}$ & $\bar{F}$ & $\bar{G}$ & $\mathrm{H}$ & $I$ & 1 & 2 & 3 & 4 & 5 & 6 & 7 & 8 & 9 & 10 & 11 & 12 & 13 & 14 & 15 & 16 & 17 & 18 & 19 & 20 & 21 & 22 & 23 & 24 & 25 & 26 \\
\hline A & E.C. & 7,24 & ,84 & ,12 & ,51" &, 22 & , 10 &,- 03 & -15 & $34^{*}$ &,- 14 & ,01 &,- 03 & ,06 &, 12 & , oo & ,08 & ,s9** &, $31^{\circ}$ & ,14 & ,08 &, 12 &,- 02 &, $32^{\circ}$ &, 15 & $\begin{array}{l}, 06 \\
\end{array}$ & ,00 & , $27 !$ &, 15 & , 13 & , 13 &, 25 & 19 & $\begin{array}{l}, 09 \\
, 0\end{array}$ &,- 14 & ,13 &, $33^{*}$ \\
\hline B & E.E. & 7,11 & 1,77 & &, $91^{*}$ &,- 07 & , 10 &,- 11 &, 13 &, 06 & ,02 &, $43^{*}$ &, 18 &, 21 & , oo &, 02 & ,09 &, 22 & ,09 & ,16 &, 13 &, 23 & ,11 &, $26^{\prime}$ &,- 03 & ,03 & ,08 &, 14 & , 03 & , 10 &, 15 & ,06 &, 12 &,- 20 &,- 10 &, 11 &, 20 \\
\hline c & E.F & 14,38 & 2,06 & & & ,08 & ,17 &,- 05 & ,11 & ,20 & ,03 &, $38^{* *}$ & ,17 &, 23 & ,07 &, 08 &, 12 &, $36^{*}$ &, 23 &, 23 & ,16 & , $26^{\prime}$ & ,1s &, $37^{*}$ & ,04 & ,06 &, 11 &, $26^{\prime}$ &, 13 &, 18 &, 21 &, 16 &, 22 &,- 14 &,- 13 &, 16 &,$s 3^{\circ}$ \\
\hline D & G & 27,57 & 4,15 & & &, 82 & ,29. & ,13 &, 02 & ,05 &,- 15 & , oo & ,02 &,- 08 &,- 18 &,$- 28 i^{\prime}$ &,- 24 &, 04 &, 15 & ,02 &,- 07 & ,08 &,- 24 &, 13 &, 12 &,- 04 &,- 04 &,- 02 &, 21 & -14 & -12 &, 01 &,- 11 & -,08 &,$- 25 !$ &, 15 &, 03 \\
\hline E & $\mathrm{BF}_{1}$ & 58,17 & 9,55 & & & &, 93 &, $35^{*}$ & ,49** & ,19 &,$s 7^{*}$ &, $48^{* *}$ &, $81^{\circ}$ & ,28! & ,28! &, 22 & ,18 &, $26^{\prime}$ &, 15 & ,49** & ${ }_{44}{ }^{*}$ & ,40"* &, $30^{*}$ &, $46^{*}$ &, $59^{* *}$ &, $47^{*}$ & ,29! & , $27 !$ &, $27 !$ &, 19 & ,15 &, $37^{*}$ & ,08 &, $30^{\circ}$ & , 27: &, $34^{\circ}$ &, $88^{*}$ \\
\hline $\mathrm{F}$ & BF2 & 53,02 & 6,96 & & & & &, 85 &, $34^{\circ}$ &, $33^{\circ}$ & ,27! &, $40^{* *}$ &, $955^{\prime}$ &, 20 &, $32^{*}$ &,$s 8^{\circ}$ &, $39{ }^{*}$ &, $85^{\circ}$ &, $29^{*}$ & $45^{\circ *}$ &, $47^{*}$ & , 288', &, $34^{*}$ &, 14 &, $35^{*}$ &, $95^{\circ}$ &, $38^{* *}$ &, $35^{*}$ &, $38^{*}$ &, $32^{*}$ &, $27 !$ &, $38^{* *}$ &, 02 &, 21 &, 17 &, $30^{\circ}$ &, $35^{\circ}$ \\
\hline G & BF3 & 55,78 & 5,72 & & & & & &, 76 &, $25^{\prime}$ &, $43^{*}$ &, $37^{\circ}$ & ,29 &, 21 &, 12 &, 19 &, 18 & ,29' &, 06 &,$s 4^{*}$ &, $31^{\circ}$ &, 23 &, $26^{\prime}$ & $46^{*}$ &,$s 9^{* *}$ &, $29^{\circ}$ &, $30^{\circ}$ & ${ }_{, 26}$ & ,08 &, $85^{*}$ &, $27 !$ &, $41^{*}$ &, 18 &, $27^{1}$ & $, 27:$ &, $37^{\circ}$ &,${ }_{, 3} 1^{\circ}$ \\
\hline н & BF4 & 55,37 & 7,82 & & & & & & &, 86 &, 19 &, 23 &,$s s^{\circ}$ & ${ }_{, 41}{ }^{*}$ &, $37^{*}$ &, $34^{\circ}$ & $40^{*}$ &, $7 q^{*}$ &, $68^{* *}$ &, $277^{\prime}$ & ,22 &,${ }_{, 5}{ }^{*}$ &, $40^{* *}$ & $49^{*}$ &,$s 1^{\circ}$ &, 21 &, $36^{*}$ &, $59^{* *}$ &, $25^{\prime}$ &, $50^{*-*}$ &, $55^{\circ *}$ & $43^{*}$ &, $34^{*}$ & ,03 &, 19 &, $47^{*}$ &, $63^{*}$ \\
\hline I & $\mathrm{BF} 5$ & 53,22 & 8,13 & & & & & & & & 82 &, $1^{*}$ &, 266 & $46^{*}$ &, $55^{*}$ &, $43^{*}$ &, $29^{\circ}$ &, $25{ }^{\prime}$ & ,14 &, $42^{* *}$ &, $34^{\circ}$ & ,19 &, $45^{*}$ &, $88^{*}$ &, $42^{*}$ & $40^{* *}$ &, $41^{*}$ &,$s 2^{*}$ &, $26^{\prime}$ &, $42^{* *}$ &, $48^{*}$ &, 21 & ,266 &, $48^{* *}$ &, $40^{*}$ &, 13 &,$s 7^{\circ}$ \\
\hline 1 & $\mathrm{CO}_{0}$ & 15,13 & 2,79 & & & & & & & & & , & $46^{*}$ &, $43^{*}$ & ,28 &, $42^{* *}$ & $40^{*}$ & $46^{*}$ &, 19 & $45^{* \prime}$ &, 26 &, $45^{*}$ &, $4 s^{*}$ &, $40^{*}$ &, $35^{\circ}$ &, $47^{*}$ &, $43^{*}$ &, $54^{* *}$ &, 15 &, $36^{\circ}$ &, $44^{*}$ &,$s^{*}$ & 29 & , &, 11 &, 19 &, $52^{*}$ \\
\hline 2 & $\mathrm{Co} 2$ & 16,50 & 2,18 & & & & & & & & & &, 67 &, $68^{*}$ &, $48^{* *}$ &, $54^{* *}$ &, $5^{* *}$ & ${ }_{, 411^{*}}$ & $49^{*}$ &, $48^{* \prime}$ &, $88^{*}$ &, $77^{*}$ &, $54^{* *}$ &, $42^{*}$ &, $58^{* *}$ &, $55^{* *}$ &, $71^{*}$ &, $59^{* *}$ &, 16 &, $65^{* *}$ & $68^{*}$ &, $54^{* *}$ &, $52^{* *}$ &, 10 & $34^{\circ}$ &, 13 &, $65^{*}$ \\
\hline 3 & Cos & 16,63 & 2,40 & & & & & & & & & & &, 75 &, $55^{* *}$ &, $51^{*}$ &, $46^{*}$ &, $51^{* *}$ &, $49^{*}$ &, $88^{*}$ &, $38^{* *}$ &, $66^{* *}$ &, $55^{* *}$ &, $48^{* *}$ &, $46^{* *}$ &, $54^{* *}$ &, $55^{* *}$ &, $72^{*}$ &, 25 &, $71^{*}$ &, $75^{*}$ & ,42"* & ,61* &, 19 &, $37^{*}$ & ,09 &, $70^{* *}$ \\
\hline 4 & $\mathrm{CO} 4$ & 20,89 & 4,07 & & & & & & & & & & & &, 78 &, $68^{*}$ &, $55^{* *}$ &, $49^{*}$ &, $42^{*}$ &, $68^{*}$ & ${ }_{, 64^{*}}$ &, $45^{*}$ &, $72^{* *}$ & $46^{*}$ &, $49^{* *}$ &, $64^{* *}$ &, $68^{*}$ &, $50^{* *}$ &, $43^{* *}$ &, $62^{* *}$ & ${ }_{, 62^{*}}$ &, $42^{* *}$ &, $50^{\circ *}$ &, $55^{* *}$ &, $54^{*}$ & ,os &, $52^{*}$ \\
\hline . & $\mathrm{CO} 5$ & 13,02 & 3,68 & & & & & & & & & & & & &, 89 &, $78^{*}$ &,$s 8^{*}$ &, $43^{*}$ &, $55^{\mu *}$ &, $58^{* *}$ &, $60^{-*}$ &, $85^{*}$ &, $32^{*}$ &, $49^{* *}$ &, $65^{* *}$ & $80^{* *}$ &, $55^{* *}$ &, $27 !$ & $69^{* *}$ &, $64 *$ & $43^{*}$ &, $60^{* *}$ & $42^{* *}$ &, $59^{* *}$ &,- 19 &, $42^{*}$ \\
\hline 6 & $\mathrm{Cog}_{0}$ & 28,91 & 5,41 & & & & & & & & & & & & & & 79 & ${ }_{42} *$ &, $5_{3}^{* *}$ & $43^{* *}$ & $.60^{\circ}$ & $66^{* *}$ &, $76^{*}$ &, $39{ }^{\circ}$ &, $50^{* *}$ & $69^{* *}$ & $.75^{* *}$ & $.57^{* *}$ & 16 & $70^{* *}$ &, $58^{*}$ & ${ }_{58}{ }^{* *}$ & $.58^{* *}$ &, 281 & $48^{*}$ & 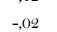 & $.51^{*}$ \\
\hline 7 & $\mathrm{C} 07$ & 32,59 & 4,81 & & & & & & & & & & & & & & &, 75 &, $60^{\circ *}$ &, $86^{*}$ &, $30^{\circ}$ &, 54 &, $32^{\circ}$ &, $47^{*}$ &, 23 &, $38^{* *}$ &, $36^{\circ}$ &, $71^{*}$ & 18 &, $48^{*}$ & ,66" &, $52^{* *}$ & ${ }_{, s 1^{\circ}}$ & ,06 & ,02 &, $43^{*}$ &, $77^{*}$ \\
\hline 8 & Cos & 32,65 & 4,25 & & & & & & & & & & & & & & & &, 64 &, $47^{*}$ & $36^{*}$ &, $67^{* *}$ & ,5s" & $46^{*}$ & $46^{*}$ & 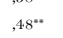 &, $42^{*}$ &, $57^{* *}$ & 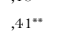 &, $54^{*}$ &, $51^{*}$ &,$x^{4}$ &, 52. & , & 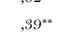 &, $47^{*}$ &, $58^{*}$ \\
\hline 9 & $\mathrm{C}_{09}$ & 20,46 & 3,38 & & & & & & & & & & & & & & & & &, 60 &, $60^{* *}$ &, $47^{*}$ &, $66^{* *}$ &, $52^{*}$ & ${ }_{, 61}{ }^{*}$ &,${ }_{, 71 *}^{*}$ &, $62^{* *}$ & ${ }_{, 41}{ }^{*}$ &, $58^{* *}$ &, $49^{* *}$ & $45^{*}$ &, $35^{\circ}$ & $\begin{array}{l}, 027 \\
{ }_{41} *\end{array}$ & 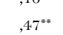 &, $59^{* *}$ &, 21 & $45^{\circ}$ \\
\hline 10 & $\mathrm{C}_{10}$ & 32,59 & 3,83 & & & & & & & & & & & & & & & & & &, 64 &,${ }_{, 51}{ }^{*}$ &, $60^{* *}$ &, $35^{\circ}$ &, $57^{* *}$ &, $57^{*}$ &, $57^{* *}$ &, $277^{\prime}$ &, $47^{* *}$ &, $52^{* *}$ &,$s 2^{*}$ &, $43^{*}$ & ,40** &, $55^{* *}$ &, $45^{*}$ & ,05 &, $3 s^{*}$ \\
\hline 11 & $\mathrm{C}_{11}$ & 33,24 & 4,20 & & & & & & & & & & & & & & & & & & &, 79 &, $59^{* *}$ &, $50^{* *}$ &, $65^{* *}$ &, $65^{* *}$ &, $67^{* *}$ &, $62^{*}$ &, $97 !$ &, $57^{*}$ &, $63^{*}$ &, $57^{*}$ & ,60" &, 21 &, $36^{*}$ & ${ }_{, 26}$ &, $67^{*}$ \\
\hline 12 & $\mathrm{C}_{12}$ & 19,35 & 4,27 & & & & & & & & & & & & & & & & & & & &, 76 &, $44^{*}$ &, $57^{\circ *}$ &, $66^{* *}$ &, $72^{* *}$ & ${ }_{, 566^{*}}$ &, $37^{*}$ &, $73^{* *}$ & ${ }_{, 63 *}$ &,$s 3^{*}$ &, $54 * *$ &, $47^{* *}$ & $63^{* *}$ &, 07 &, $42^{* *}$ \\
\hline 13 & $\mathrm{C}_{13}$ & 22,70 & 3,06 & & & & & & & & & & & & & & & & & & & & & 61 &, $56^{*}$ &, $55^{* *}$ & $46^{*}$ &,${ }^{4} *^{* *}$ &, $25^{\prime}$ &, $56^{* *}$ &, $50^{\circ *}$ &, $544^{* * *}$ & $46^{*}$ & 12 & 24 &, $29^{\circ}$ &, $63^{*}$ \\
\hline $\begin{array}{l}13 \\
14\end{array}$ & $\mathrm{C}_{14}$ & $\begin{array}{l}12,10 \\
16,20\end{array}$ & $\begin{array}{l}3,060 \\
2,66\end{array}$ & & & & & & & & & & & & & & & & & & & & & & , & 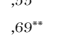 & $\begin{array}{l}x^{406} \\
, 59^{-*}\end{array}$ & 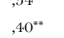 & $\begin{array}{l}20 \\
{ }_{, 42}^{*}\end{array}$ & $\begin{array}{l}., 56 \\
, 50^{* *}\end{array}$ & 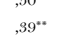 & 年, & 年, & , & 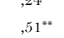 & , 29 & $\begin{array}{l}x^{6.03} \\
x^{*}\end{array}$ \\
\hline 15 & $\mathrm{C}_{15}$ & 23,17 & , & & & & & & & & & & & & & & & & & & & & & & &, 74 &, $69^{* *}$ &, $59^{* *}$ &, $38^{*}$ &, $59^{*-}$ &, $54^{*}$ &, $60^{\circ}$ &, $50^{\circ *}$ &, $47^{*}$ &, $57^{*}$ &, 10 &, $57^{*}$ \\
\hline 16 & $\mathrm{C}_{16}$ & 21,43 & $\begin{array}{l}3,36 \\
3,6\end{array}$ & & & & & & & & & & & & & & & & & & & & & & & &, 70 &, $50^{* *}$ & ,26 &, $75^{*}$ & $66^{*}$ &,${ }_{54}$ & ${ }_{62}$ &, $35^{\circ}$ &, $51 *$ & -,os &, $52 * *$ \\
\hline 17 & $\mathrm{C}_{17}$ & 16,8 & 2,30 & & & & & & & & & & & & & & & & & & & & & & & & &, 71 &, 12 &, $64^{* *}$ &, $79^{*}$ &, $56^{*}$ & ${ }_{51}{ }^{*}$ & 06 & .24 &, 20 & $82^{*}$ \\
\hline 18 & C18 & 16,65 & 2,56 & & & & & & & & & & & & & & & & & & & & & & & & & &, 61 &, 19 & ,19 & -,01 &, 21 &, $30^{\circ}$ &, $47^{*}$ &, 271 & ,19 \\
\hline 19 & $\mathrm{C}_{19}$ & ${ }_{21,61}$ & 3,21 & & & & & & & & & & & & & & & & & & & & & & & & & & &, 72 & $72^{*}$ & ${ }_{61}{ }^{*}$ &, $57^{* *}$ &,, $5^{\circ}$ &, $48^{* *}$ &, 10 &, $61^{*}$ \\
\hline 20 & $\mathrm{C}_{20}$ & 15, & $\begin{array}{l}\text { o, } \\
2,31\end{array}$ & & & & & & & & & & & & & & & & & & & & & & & & & & & & $\begin{array}{l}, 12 \\
, 79\end{array}$ & 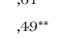 & 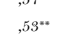 & , & , & 4 &, $66^{*}$ \\
\hline 21 & $\mathrm{C}_{21}$ & 34,41 & 2,97 & & & & & & & & & & & & & & & & & & & & & & & & & & & & &, 55 & ${ }_{41}{ }^{* *}$ &, 15 & ${ }_{91}$ &, 16 &, $62^{*}$ \\
\hline 22 & C29 & 21,1 & 3,01 & & & & & & & & & & & & & & & & & & & & & & & & & & & & & & 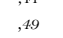 & 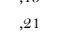 & 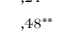 &,- 04 & 1. \\
\hline 23 & C2s & 20,9 & 5,29 & & & & & & & & & & & & & & & & & & & & & & & & & & & & & & &, 82 &, $57^{* *}$ & 9 & 7 \\
\hline $\begin{array}{l}23 \\
24\end{array}$ & $\mathrm{C}_{24}$ & $\begin{array}{l}00,93 \\
10,96\end{array}$ & $\begin{array}{l}0,29 \\
3,99\end{array}$ & & & & & & & & & & & & & & & & & & & & & & & & & & & & & & & & 68 & , 05 & 16 \\
\hline 25 & $\mathrm{C}_{2}$ & 34 & 4,27 & & & & & & & & & & & & & & & & & & & & & & & & & & & & & & & & &, 73 & $8^{* *}$ \\
\hline 26 & C26 & 23,15 & 3,11 & & & & & & & & & & & & & & & & & & & & & & & & & & & & & & & & & & 71 \\
\hline
\end{tabular}

Nota: En la diagonal principal se muestran los valores Alfa de Cronbach. E.C.: Ev. Continua; E.E.: Examen; EF.: Ev. Final; G: Fac. G; BF 1: Ajuste Emoc. BF2: Extraversión; BF3: Cordialidad: BF 4: Responsabilidad; BF 5: Apert. a la Experiencia; Co 1: Cap. de Aprendizaje; Co2: Utilización de Conoc.; Co3: Cap. de Análisis y Síntesis; Co4: Hab. de Búsqueda y Gestión de info.; Co5: Innovación; C06: Liderazgo; C07: Interés por la Calidad; C08: Ori. al Cliente; Co9: Ori. a Resultados; C10: Trabajo en Equipo; C11: Comunicación; C12: Iniciativa; C13: Seguridad en sí mismo; C14: Autocontrol; C15: Flexibilidad-Gestión del Cambio; C16: Negociación-Persuasión; C17: Planificación y Organización; C18: Hab. Informáticas Básicas; C19: Reso. de Problemas; C20: Toma de Decisiones; C21: Asertividad; C22: Cap. de crítica y autocrítica; C23: Conocimiento y Valoración de la Diversidad; C24: Resp. Social; C25: Resp. Laboral; C26: Hab. trabajo Autonmo. M: Media; DT: desviación típica. * significativa a nivel 0,01 (bilateral) ** significativa a nivel 0,05 (bilateral).; significativa a nivel $<0,1$. 
Finalmente, con el objetivo de ver el incremento de varianza explicada sobre las variables de personalidad e inteligencia, solo pudimos trabajar con la Evaluación Continua ya que de entre las variables de personalidad e inteligencia únicamente el factor de Responsabilidad correlacionaba con dicho criterio. En el primer paso de la regresión jerárquica se incluyó la Responsabilidad y en el segundo paso se incluyó la competencia C07: Interés por la Calidad. Los resultados muestran que al introducir la Responsabilidad se produce un incremento de varianza explicada $\Lambda R^{2}=0,117$ significativo $\left(\mathrm{F}_{1,41}=5,438\right.$; Sig. 0,025) y que al introducir en el segundo paso la Co7: Interés por la Calidad se produce un incremento de varianza explicada de 0,045 que no resulta ser significativo $\left(\mathrm{F}_{1,40}=2,132 ; \mathrm{Sig}=0,152\right)$.

\section{Discusión}

En este trabajo se presenta un cuestionario para la evaluación de las competencias transversales en universitarios respondiendo a una necesidad no resuelta derivada de la implantación de programas basados en competencias genéricas para universitarios

Los análisis realizados muestran que el cuestionario desarrollado manifiesta unas propiedades psicométricas aceptables en línea con los estándares al uso. El cuestionario es en general fiable, muestra una adecuada validez de contenido y presenta una estructura latente coherente con el modelo sustantivo de referencia. A pesar de ello, hay que prestar especial atención a las escalas de Asertividad y Capacidad de Crítica y Autocrítica, ambas con índices pobres en fiabilidad y validez de contenido. Estos datos deberían ser analizados en mayor profundidad de cara a averiguar las razones del funcionamiento incorrecto de las escalas, si bien podrían estar relacionados con posibles sesgos de deseabilidad social debido a los contenidos que evalúan ambas escalas.

En lo que respecta a la fiabilidad de las escalas se ha podido observar cómo todas ellas (a pesar de que algunas solo tienen cinco elementos) mantienen unos indicadores de fiabilidad satisfactorios (por encima de 0,70 ) a excepción de las dos competencias señaladas. Un efecto similar sucede para la validez de contenido, en el que para casi todas las escalas se alcanzan valores superiores a .80. Aun así, debe señalarse la existencia de tres escalas para las que se obtienen índices de congruencia bajos: Asertividad, Capacidad de Crítica y Autocrítica y Habilidad para Trabajar de Forma Autónoma.

En lo que respecta a la validez factorial, el porcentaje de varianza explicada $(64,74 \%)$ es alto, lo que da muestra de un buen comportamiento del modelo factorial propuesto. Además, la estructura de cuatro factores o dimensiones (competencia para la Innovación, competencia para la Acción, competencia para la Interacción y competencia para el Compromiso Social) es comparable con la propuesta del Proyecto Tuning y sus tres dimensiones generales (competencias Instrumentales, Interpersonales y Sistémicas). A pesar de que en nuestro estudio hallamos cuatro factores, creemos que existen similitudes entre nuestro factor de competencias para la Acción y las competencias Instrumentales del Proyecto Tuning, así como entre nuestro factor de competencias para la Interacción y las competencias Interpersonales del Proyecto Tuning. Del mismo modo, nuestros factores de competencias para la Innovación y para el Compromiso Social muestran amplias similitudes con algunas de las competencias incluidas en las competencias Sistémicas del Proyecto Tuning. 
$\mathrm{Al}$ analizar los estadísticos descriptivos llaman la atención las intercorrelaciones entre las 26 competencias evaluadas. El hecho de que la gran mayoría de ellas se relacionen de forma significativa con el resto podría interpretarse como una señal de que se están midiendo constructos relacionados entre sí. Esto resulta lógico si tenemos en cuenta que las competencias genéricas pueden verse como diferentes aspectos de una capacitación general de los estudiantes universitarios, lo que sigue la línea propuesta por algunos autores según la cual los perfiles universitarios basados en competencias genéricas deberían presentar un alto grado de coherencia interna y unidad (Cabrerizo, Rubio y Castillo, 2008).

Adicionalmente, los resultados obtenidos en el segundo estudio suponen evidencia inicial acerca de la validez predictiva del CCT respecto de los resultados académicos de los alumnos. En cualquier caso debe ser señalado que no todas las competencias contribuyen a explicar el rendimiento académico, lo cual puede ser debido a que éste ha sido evaluado para una asignatura específica de una determinada titulación (en la que se imparten y evalúan una serie de conocimientos específicos para los que unas competencias transversales serán más relevantes que otras) y, además, se han contemplado exclusivamente los resultados del alumno expresados en la evaluación del profesor mediante el examen y la evaluación continua (dejando por tanto de lado aspectos del desempeño del alumno que son relevantes para la consecución de los objetivos académicos). En este sentido las competencias que han mostrado ser relevantes para predecir el rendimiento académico en la asignatura son: Interés por la Calidad (para la Evaluación Continua) y Capacidad de Aprendizaje (para la Evaluación Examen y Evaluación Final). Por otro lado, atendiendo a la personalidad y la inteligencia, nuestros resultados muestran que únicamente la Responsabilidad muestra una relación significativa con el rendimiento académico (con la puntuación en Evaluación Continua). En este sentido es necesario tener en cuenta que la personalidad y el rendimiento académico podrían estar relacionadas de forma no lineal (Furnham y Chamorro, 2004; Poropat, 2009).

Los estudios presentados tienen algunas limitaciones que inciden en la interpretación del trabajo. En lo que se refiere a las cualidades métricas del cuestionario, las características de la muestra escogida para los dos estudios del trabajo hacen que los hallazgos no sean generalizables por completo. Esto es debido a que toda la muestra proviene de una misma universidad, en el primer estudio, y a que su número no es lo suficientemente amplio en el segundo: pocos alumnos, de una asignatura en una sola Universidad, y con un criterio en el que únicamente se contemplan los resultados de la evaluación de la asignatura. Ello viene a indicarnos que la generalización de los resultados a otras asignaturas y muestras debe realizarse con extrema precaución. Por ello, posteriores trabajos deben ser desarrollados para ampliar tanto el rango de participantes (diferentes asignaturas, diferentes cursos, diferentes Universidades) como los criterios de valoración académica utilizados. Por otro lado, en la medición de la validez de constructo se llevó a cabo un análisis factorial exploratorio, por lo que debería continuarse el análisis con el consiguiente análisis factorial confirmatorio. Por otro lado, la selección de las variables de personalidad e inteligencia en el segundo estudio puede resultar discutible. Si bien tratamos de utilizar el modelo del Big Five para la personalidad y el de Factor G para la inteligencia por ser las propuestas más empleadas y difundidas en la literatura, no son ni mucho menos los únicos modelos existentes para estos dos constructos psicológicos. Estos dos modelos suponen una aproximación general a las variables de personalidad e 
inteligencia, dado que ambos proponen factores generales. A pesar de que la elección de factores generales permite la comparación de los resultados, pueden estar oscureciendo relaciones más finas entre las variables que se mostrarían con modelos más detallados (Poropat, 2009). Algo similar ocurre con la elección de las variables criterio en el segundo estudio: la calificación académica resulta igualmente una variable general que puede hacernos perder detalle de algunas relaciones más concretas entre el desempeño académico y las competencias.

En este sentido, dos son las líneas de investigación prioritarias: el desarrollo de estudios que permitan refinar la medida de las competencias transversales de los alumnos universitarios, y el desarrollo de estudios específicos en los que se explore la capacidad de las competencias transversales para explicar y predecir diferentes comportamientos y criterios académicos.

Estos resultados tienen interés también desde el punto de vista aplicado, ya que ofrecen a los docentes y a las instituciones una herramienta útil para apreciar las competencias transversales de sus alumnos. Como se extrae del Proyecto Tuning, el desarrollo de competencias transversales es de la mayor relevancia para los estudiantes universitarios y para las escuelas y facultades, debido a que otorga a los estudiantes unos recursos esenciales para su inserción laboral. En este sentido, aun teniendo en cuenta las limitaciones expresadas, las evidencias psicométricas presentadas señalan como el CCT puede ser utilizado en contextos aplicados. Tanto para valorar las posibles dificultades y puntos fuertes de los alumnos en su desempeño académico: el CCT al centrarse en comportamientos permite determinar cuáles de ellos son ineficientes para un alumno específico y establecer pautas de acción y mejora; como para valorar de manera conjunta la evolución de las competencias transversales en un determinado centro o institución. Ello sin duda es relevante desde el punto de vista de la necesidad por parte de las universidades de adoptar los dictámenes que se exigen desde el Proyecto Tuning y el EEES para implantar modelos de competencias en sus perfiles y currículos.

Finalmente, es necesario señalar la importancia de entender qué factores facilitan y explican el desempeño académico de los estudiantes y su futura inserción laboral, lo que implica una visión de los perfiles académicos que contemplan las competencias transversales y que supone un importante valor añadido para las facultades, escuelas y sus docentes en su intento de otorgar a los alumnos los conocimientos, habilidades y actitudes necesarios para hacer frente a su futuro como profesionales. Para ello, se requiere de modelos e instrumentos de evaluación que permitan obtener una medida válida y fiable de dichas competencias.

\section{Referencias}

Accenture. y Universia. (2007). Las competencias profesionales en los titulados. Contraste y diálogo universidad $\quad y \quad$ empresar Recuperado de http://www.accenture.com/Countries/Spain/About_Accenture/LasEmpresa.htm

Aguado, D. y Lucía, B. (s.f.). Guía breve de eValue. Informe técnico de investigación. Documento sin publicar.

Aguado, D., Lucia, B., Ponte, G. y Arranz, V. (2008). Análisis inicial de las propiedades psicométricas del cuestionario BFCP Internet para la evaluación de Big-Five. Revista Electrónica de Metodología Aplicada, 13(2), 1-15. 
Akkermans, J., Schaufeli, W. B., Brenninkmeijer, V. y Blonk, R. W. B. (2013). The role of career competencies in the job demands-resources model. Journal of Vocational Behavior, 83(3), 356-366. doi:10.1016/j.jvb.2013.06.011

Atkinson, H. H., Lambros, A., Davis, B. R., Lawlor, J. S., Lovato, J., Sink, K. M. y Williamson, J. D. (2013). Teaching medical student geriatrics competencies in 1 week: An efficient model to teach and document selected competencies using clinical and community resources. Journal of the American Geriatrics Society, 61(7), 1182-1187. doi:10.1111/jgs.12314

Bartram, D. (2005). The great eight competencies: A criterion-centric approach to validation. Journal of Applied Psychology, 90(6), 1 185-1203. doi:10.1037/002 1-9010.90.6.1185.

Blömeke, S., Zlatkin-Troitschanskaia, O., Kuhn, C. y Fege, J. (Eds.) (2013). Modeling and measuring competencies in higher education. Berlin: SensePublishers.

Boam, R. y Sparrow, P. (1992). The rise and rationale of competency-based approaches. En R. Boam y P. Sparrow (Eds.), Designing and achieving competency (pp. 3-15). Londres: McGraw Hill.

Boyatzis, R. (1982). The competent manager. Nueva York, NY: Wiley.

Cabrerizo, J., Rubio, M. J. y Castillo, S. (2008). Programación por competencias. Formación y práctica. Madrid: Pearson Educación.

Costa, P. T. y McCrae, R. R. (1985). The NEO personality inventory manual. Odessa, FL: Psychological Assessment Resources.

Costa, P. T. y McCrae, R. R. (1992). The revised NEO personality inventory (NEO-PI-R) and NEOfive-factor inventory (NEO-FFI) professional manual. Odessa, FL: Psychological Assessment Resources.

Elosua, P. y Zumbo, B. (2008). Coeficientes de fiabilidad para escalas de respuesta categórica ordenada. Psicothema, 20(4), 896-901.

Feltham, R. (1992). Using competencies in selection and recruitment. En R. Boam y P. Sparrow (Eds.), Designing and achieving competency (pp. 89-103). Londres: McGraw Hill.

Furnham, A. y Chamorro, T. (2004). Personality and intelligence as predictors of statistics examinations grades. Personality and Individual Differences, 37, 943-955. doi:10.1016/j.paid.2003.10.016

García, M. (2011). Una revisión constructiva de la gestión por competencias. Anales de Psicología, 27(2), 473-497. doi:10.6018/123101

Gómez, J. M. y Borrás, F. (2006). Competencias profesionales en los titulados UMH. Elche: Universidad Miguel Hernández.

González, M. (2006). Currículo basado en competencias: una experiencia en educación universitaria. Educación y Educadores, 9(2), 95-117.

González, J. y Wagenaar, R. (2003). Tuning educational estructures in Europe. Bilbao: Universidad de Deusto.

Jung, H. Y. (2015). The study on essential competencies for university students in consideration of university specialization and major: focusing on hotel and tourism management. Journal of Digital Convergence, 13(10), 19-32. doi:10.14400/jdc.2015.13.10.19

Khairullina, E. R., Pochinova, T. V., Khisamiyeva, L. G., Sakhipova, Z. M., Fedorova, L. V., Ablyasova, A. G. y Aksenova, N. N. (2015). The competences model of competitive process engineer. Journal of Sustainable Development, 8(3), $250-266$. doi:10.5539/jsd.v8n3p250 
Kurz, R. y Bartram, D. (2002). Competency and individual performance: Modelling the world of work. En T. Robertson, M. Callinan y D. Bartram (Eds.), Organizacional effectiveness. The role of psychology (pp. 227-255). Chichester: Routledge.

Latham, G. P. y Wexley K. N. (1981). Increasing productivity through performance appraisal. Reading, MA: Addison-Wesley.

Lawler, E. E. (1994). From job-based to competency-based organization. Journal of Organizacional Behaviour 15, 3-15. doi:10.1002/job.4030150103.

Lozano, L., García-Cueto, E. y Muñiz, J. (2008). Effect of the number of response categories on the reliability and validity of rating scales. Methodology, 4(2), 73-79. doi:10.1027/16142241.4 .2 .73

Lucia, A. D. y Lepsinger, R. (1999). Competency models. Pinpointing critical success factors in organizations. San Francisco, CA: Jossey Bass Pfeiffer.

McClelland, D. C. (1973). Testing for competence rather than for intelligence. American Psychologists, 28, 1-14. doi:10.1037/h0034092

Murphy, K., Martin, C. y García, M. (1982). Do behavioral observation scales measure observation? Journal of Applied Psychology, 67(5), 562-567. doi:10.1037/0021-9010.67.5.562

Nikolau, I. (2003). The development and validation of a measure of generic work competencies. International Journal of Testing, 3(4), 309-319. doi:10.1207/S15327574IJT0304_1

Nybo, G. (2004). Personnel development for dissolving jobs: Towards a competency-based approach? International Journal of Human Resource Management, 15(3), 549-564. doi:10.1080/0958519042000181250.

Osterlind, S. J. (1989). Constructing test items. Boston, MA: Kluwer Academic Publishers.

Palmer, A., Montaño, J. y Palou, M. (2009). Las competencias genéricas en la educación superior. Estudio comparativo entre la opinión de empleadores y académicos. Psicothema, 21(3), 433438.

Poropat, A. (2009). A meta-analysis of the five-factor model of personality and academic performance. Psychological Bulletin, 135(2), 322-338. doi:10.1037/a0014996

Rieckmann, M. (2012). Future-oriented higher education: Which key competencies should be fostered through university teaching and learning? Futures, 44(2), 127-135. doi:10.1016/j.futures.2011.09.005

Robertson, I. y Kinder, A. (1993). Personality and job competentes: The criterion-related validity of some personality variables. Journal of Occupational and Organizational Psychology, 66, 225-244. doi: 10.1111/j.2044-8325.1993.tb00534.x

Robertson, I., Gibbons, P., Baron, H., MacIver, R. y Nyfield, G. (1999). Understanding management performance. British Journal of Management, 1O(5), 5-12. doi:10.1111/14678551.00107

Rovinelli, R. y Hambleton, R. K. (1977). On the use of content specialists in the assessments of criterion-referenced test item validity. Dutch Journal of Educational Research, 2, 49-60.

Schmeiser, C. B. y Welch, C. J. (2006). Test development. En R. L. Brennan (Ed.), Educational measurement (pp. 45-76). Westport, CT: Praeger Publishers.

Shek, D. T. y Sun, R. C. (2012). Promoting psychosocial competencies in university students: Evaluation based on a one-group pre-test/post-test design. International Journal on Disability and Human Development, 11(3), 229-234. doi:10.1515/ijdhd-2012-0039 
SHL Group. (1993a). Inventory of management competencies: Manual and user's guide. Thames Ditton: SHL Group plc.

SHL Group. (1993b). OPQ concept model: Manual and user's guide. Thames Ditton: SHL Group plc.

SHL Group. (1994). Perspectives on managerial competencies: User's manual. Thames Ditton: SHL Group plc.

SHL Group. (1997). Customer contact: Manual and user's guide. Thames Ditton: SHL Group plc.

SHL Group. (1999a). OPQ32: Manual and user's guide. Thames Ditton: SHL Group plc.

SHL Group. (1999b). Work styles questionnaire, version n: Manual and user's guide. Thames Ditton: SHL Group plc.

Solanes, A., Núñez, R. y Rodríguez, J. (2008). Elaboración de un cuestionario para la evaluación de competencias genéricas en estudiantes universitarios. Apuntes de Psicología, 26(1), 35-49.

Sparrow, P. (1997). Organizational competencies: Creating a strategic behavioural framework for selection and assessment. En N. Anderson y P. Herriot (Eds.), International Handbook of Selection and Assessment (pp. 343-368). Chichester: Wiley.

Sparrow, P. y Bognanno, M. (1993). Competency requirement forecasting: Issues for international selection and assessment. International Journal of Selection and Assessment, 1 , 50-58. doi:10.1111/j.1468-2389.1993.tboo083.x

Spencer, L. M. y Spencer, S M. (1993). Competence at work, models for superior performance. New Cork: Wiley.

Tea Ediciones. (2008). SOSIA. Recuperado de http://web.teaediciones.com/SOSIA--GESTIONPOR-COMPETENCIAS.aspx

Tea Ediciones. (2011). CompeTEA. Recuperado de http://web.teaediciones.com/COMPETEA.aspx

Tett, R., Guterman, H., Bleier, A. y Murphy, P. (2000). Development and content validation of a "hyperdimensional" taxonomy of managerial competence. Human Performance, 13(3), 205251. doi:10.1207/S15327043HUP1303_1

Vivel-Búa, M., Fernández-López, S., Lado-Sestayo, R. y Otero-González, L. (2015). ¿Cómo mejorar la asimilación de los contenidos teóricos por parte del alumnado universitario? Una aplicación del one minute paper en Contabilidad. REICE. Revista Iberoamericana sobre Calidad, Eficacia y Cambio en Educación, 13(2), 67-84.

Weng, L. (2004). Impact of the number of response categories and anchor labels con coefficient alpha and test-retest reliability. Educational and Psychologycal Measurement, 64, 956-972.

Wiersma, U. y Latham, G. (1986). The practicality of behavioral observation scales, behavioral expectation scales and trait scales. Personnel Psychology, 39, 619-628. doi:10.1111/j.17446570.1986.tboo956.x

Zhao, L., Sun, T., Sun, B. Z., Zhao, Y. H., Norcini, J. y Chen, L. (2015). Identifying the competencies of doctors in China. BMC Medical Education, 15(1), 1-8. doi:10.1186/s12909015-0495-y 


\section{Apéndice I. Modelo de 26 competencias genéricas para población universitaria.}

Contenidos de evaluación y ejemplos de ítems recuperados de López y colaboradores (2007).

Co1. Capacidad de aprendizaje. Adquirir y asimilar conocimientos por medio del estudio, de la observación o de la experiencia o construirlo de forma activa. Asimilar nuevos conceptos, conocimientos y procedimientos sin dificultad. Necesitar mucho tiempo para entender un concepto o procedimiento nuevo en profundidad.

Co2. Utilización de conocimientos. Transferir y utilizar un conocimiento o habilidad adquirida a una situación novedosa. Ante una situación nueva, detectar los elementos relacionados con los conocimientos que poseo. Aprovechar la formación que recibo.

Co3. Capacidad de análisis y síntesis. Descomponer un hecho, problema o situación en las diversas partes que lo componen, y extraer lo relevante. Identificar los elementos clave de un problema. Al preparar una presentación, distinguir el tema principal del de sus partes.

Co4. Habilidades de búsqueda y gestión de la información. Buscar activamente información y complementar los conocimientos a partir de diversas fuentes. Buscar información en múltiples fuentes para desarrollar mi trabajo (Internet, publicaciones, aplicaciones corporativas, personas que son fuentes fiables de información, etc.). Contrastar la calidad y validez de la información que obtengo acerca de un tema, por ejemplo, consultando otras fuentes o consultando a expertos.

Co5. Innovación. Proponer mejoras, acciones novedosas y soluciones innovadoras y eficaces. Proponer ideas innovadoras que suponen mejoras significativas. Salirme de lo establecido y aportar ideas diferentes.

Co6. Liderazgo. Asumir la responsabilidad de guiar a un equipo de personas, preocupándose por su desarrollo y motivándolos para alcanzar el objetivo propuesto. Liderar las reuniones de trabajo y tomar la iniciativa. Mostrar interés por liderar grupos y responsabilizarme de ello.

Co7. Interés por la calidad. Conseguir resultados de calidad excelente, estableciendo mecanismos de revisión y control del trabajo para evitar errores y dificultades. Mantener ordenado mi espacio de trabajo y localizar sin problemas dónde está cada cosa. Revisar mis tareas varias veces y en profundidad antes de darlas por finalizadas.

Co8. Orientación al Cliente. Conocer, identificar y satisfacer las necesidades de los clientes para resolver activamente sus demandas. Mantener un seguimiento de la situación de mi trabajo, con las personas que lo reciben. Tener una comunicación fluida y cordial con los receptores de mi trabajo, estableciendo un clima de confianza.

Co9. Orientación a resultados. Orientar el esfuerzo a la consecución de objetivos y resultados (parciales o finales), de la manera más eficaz y eficiente posible. Tener en cuenta la relación coste-beneficio a la hora de planificar los objetivos. Tener como prioridad alcanzar los objetivos fijados en mi trabajo.

C10. Trabajo en Equipo. Colaborar activamente con el equipo de trabajo, coordinando las tareas a realizar con todos sus componentes, compartiendo información y recursos y asumiendo las decisiones tomadas en grupo. Tener en cuenta el impacto (positivo o negativo) 
de mis actuaciones y tratar de no perjudicar al equipo. Participar activamente en las clases o en reuniones de trabajo (doy mi opinión, pregunto a los demás...).

C11. Comunicación. Expresar con claridad y precisión las ideas y los hechos, tanto verbal como por escrito. Expresar y escribir las ideas o los hechos con claridad y concision. Mantener un discurso ordenado y resaltar los aspectos más importantes del mensaje, de manera que sea lo más fácilmente entendible para el otro.

C12. Iniciativa. Actuar por iniciativa propia, idear y emprender actividades, anticiparse a los demás, desarrollar ideas. En situaciones de crisis ser rápido en reaccionar y proponer soluciones. Ofrecer mi colaboración y servicios antes de que me lo demanden.

C13. Seguridad en si mismo. Manifestar seguridad en las propias capacidades y características, asumiendo la responsabilidad de los errores y de los éxitos de manera positiva. Cuando estoy convencido de un determinado modo de actuación, confiar y persistir en ello a pesar de los obstáculos que puedan surgir. Afrontar con seguridad y confianza cualquier tipo de tarea por dificil o desconocida que esta sea.

C14. Autocontrol. Mantener el rendimiento y la calma ante situaciones estresantes o de tensión, controlando adecuadamente las emociones. Mantener mi rendimiento habitual en situaciones de estrés (acumulación de trabajo, presión, etc.). En momentos difíciles o de tensión, mantener un enfoque positivo hacia personas o situaciones (no pierdo el control, tranquilizo a otros, no me desanimo, no me bloqueo, etc.)

C15. Flexibilidad/ Gestión del cambio. Adaptarse con facilidad a nuevas situaciones o procedimientos, trabajando de forma eficaz en distintas situaciones con personas y grupos diversos valorando enfoques distintos al propio. Adaptarme sin problemas a los cambios de tarea, herramientas o tecnología. Ser capaz de trabajar con grupos de personas distintos.

C16. Negociación/ Persuasión. Conseguir llegar a acuerdos duraderos entre las diferentes partes de un conflicto de intereses, argumentando con seguridad. Ser claro y convincente en mis argumentaciones. Conseguir convertir las objeciones de mi interlocutor en posibilidades u oportunidades.

C17. Capacidad de planificación y organización. Programar las acciones, recursos y plazos necesarios para la ejecución de un proyecto o tarea. Cuando me encomiendan una tarea, estimar el tiempo que ésta me puede llevar. Definir los pasos a seguir para realizar un trabajo.

C18. Habilidades informáticas básicas. Utilizar con destreza programas informáticos como procesadores de textos, hojas de cálculo, bases de datos. Manejar sin problemas las operaciones básicas de Windows. Saber hacer las tareas que me piden utilizando una hoja de cálculo.

C19. Resolución de problemas. Analizar un problema encontrando sus causas y consecuencias para encontrar y aplicar soluciones eficaces. Identificar todas las posibles consecuencias de un problema. Ser capaz de definir las causas y consecuencias de un problema.

C20. Toma de decisiones. Actuar y decidir de manera activa, sopesando las diferentes alternativas de actuación y eligiendo la más adecuada para realizarla posteriormente. Estudiar las características de las alternativas que se me plantean para tomar una decisión. Ordenar las alternativas de solución de un problema utilizando los criterios adecuados. 
C21. Asertividad. Expresar los sentimientos, opiniones y puntos de vista con seguridad pero sin agredir ni ser agredido. A la hora de expresar mi opinión hacerlo sin agresividad y con calma, dejando hablar a los demás aunque manifestando claramente mi punto de vista. Expresar a los demás mis sentimientos.

C22. Capacidad crítica y autocrítica. Valorar objetivamente las propias capacidades y habilidades, siendo consciente de sus puntos fuertes y sus necesidades de desarrollo. Valorar de manera objetiva las capacidades de los demás proporcionando una crítica constructiva si se solicita. Aceptar las críticas cuando cometo un error. Entender que los demás puedan mejorar mis planteamientos.

C23. Conocimiento y valoración de la diversidad. Conocer, apreciar y respetar la diversidad social y cultural. Documentarme acerca de lo que ocurre o cómo se vive en otros países. Pensar que si me relaciono con personas extranjeras, podré entender los problemas interculturales y asi a frontarlos.

C24. Responsabilidad social. Asumir las consecuencias de nuestras acciones y decisiones, actuar de acuerdo con una noción de justicia social y de sentido del deber del deber para con los demás. Llevar a cabo conductas comprometidas con el medio ambiente. Seguir las normas establecidas en materia de sostenibilidad.

C25. Responsabilidad Laboral. Aceptar y asumir normas que regulan las interacciones en el mercado laboral y que generalmente quedan recogidas en el código deontológico o código ético. Cumplir cuando me comprometo con algo. No desanimarme o aburrirme ante actividades rutinarias, y mantener el ritmo de trabajo.

C26. Habilidad para trabajar de forma autónoma. Aprender, desenvolverse y trabajar por sí solo de manera responsable sin necesidad de supervisión, ayuda o apoyo permanente. Programar mis tareas ajustándome al tiempo disponible. En mis actividades, saber lo que tengo que hacer en cada momento.

\section{Breve CV de los autores}

\section{David Aguado}

Doctor en Psicología por la Universidad Autónoma de Madrid y profesor de la misma Universidad en el área de Psicología Social. Es también Investigador Senior en el Instituto de Ingeniería del Conocimiento el que ha desarrollado diferentes trabajos de aplicación de modelos de desarrollo de competencias transversales a través de sistemas informatizados en el entorno universitario. Es director del Programa de Desarrollo de Competencias Transversales UAM-ADIC mediante el que se favorece la inserción laboral de los alumnos de la universidad mediante el desarrollo de sus competencias no técnicas. ORCID ID: 0000-0001-9747-2167. Email: david.aguado@uam.es 


\section{Antonio González}

Licenciado en Psicología y consultor de formación y selección. Especialmente vinculado con la valoración de competencias de los universitarios en contextos de selección de personal ha desarrollado diferentes trabajos encaminados a dotar de herramientas que puedan ser utilizadas para valorar las competencias transversales de los universitarios. ORCID ID: 0000-0002-8545-0734. Email: agonzalezencinas@gmail.com

\section{Marta Antúnez}

Licenciada en Psicología y Máster en Dirección de Recursos Humanos por la Universidad Autónoma de Madrid. Actualmente desarrolla proyectos de selección de universitarios para puestos técnicos en grandes organizaciones. Es especialista en valoración de competencias transversales. ORCID ID: 0000-0002-4466-279X. Email: marta.antex@gmail.com

\section{Teresa de Dios}

Doctora en Humanidades y Ciencias Sociales. Master Oficial en Humanidades. Master en Recursos Humanos. Licenciada en Psicología. Diplomada en Magisterio. Profesora en el área de Administración y Dirección de empresas y Responsable de la Coordinación de Formación e Innovación docente en la Universidad Francisco de Vitoria. Es además profesora en la Cámara Oficial de Comercio e Industria de Madrid y en la Universidad Autónoma. ORCID ID: 0000-0001-7746-9307. Email: t.dedios.prof@ufv.es 